Title: Towards multi-disciplinary HIV cure research: integrating social science with biomedical research

\author{
Authors: \\ Cynthia I Grossman, $\mathrm{PhD}^{1}$, Anna Laura Ross, $\mathrm{PhD}^{2}$, Judith D. Auerbach, $\mathrm{PhD}^{3}$, Jintanat \\ Ananworanich, MD, $\mathrm{PhD}^{4}$, Karine Dubé, MPhil, DrPH Candidate ${ }^{5}$, Joseph D. Tucker, MD, $\mathrm{PhD}^{6}$, \\ Veronica Noseda, $\mathrm{MSc}^{7}$, Cristina Possas, $\mathrm{PhD}^{8}$, Dianne Rausch, $\mathrm{PhD}^{1}$, on behalf of the IAS \\ Towards an HIV Cure initiative
}

\begin{abstract}
Affiliations:
${ }^{1} 5601$ Fishers Lane Room 9G19, MSC 9831, National Institutes of Mental Health (NIMH), National Institutes of Health (NIH), Bethesda, MD, 20892, USA; Phone: +1 240627 3868; Email: grossmanc@mail.nih.gov; drausch@mail.nih.gov

${ }^{2}$ Agence Nationale de Recherche sur le SIDA (ANRS), 101 rue de Tolbiac, 75013 Paris, France; +33 15394 60 17; Email : anna-laura.ross@anrs.fr

${ }^{3}$ Center for AIDS Prevention Studies, Department of Medicine, School of Medicine, University of California, Mailcode 0886, $55016^{\text {th }}$ Street, $3^{\text {rd }}$ Floor, San Francisco, CA, 94158, USA; +1 475597 9106; Email: judithd.auerbach@gmail.com

${ }^{4}$ 6720A Rockledge Drive, Suite 400, The Henry M. Jackson Foundation for the Advancement of Military Medicine, Inc., Bethesda, MD, USA; United States Military HIV Research Program (MHRP), Bethesda, MD, 20852; USA; Phone: +1 301-500 3949; Email: jananworanich@hivresearch.org

5120 Mason Farm Road, \#2047 Genetic Medicine Building, Institute of Global Health and Infectious Diseases (IGHID), Collaboratory of AIDS Researchers for Eradication (CARE), Chapel Hill, NC, 27516, USA; Email: karine_dube@med.unc.edu

${ }^{6}$ UNC Project-China, 2 Lujing Road, Guangzhou, China, 510095; University of North Carolina at Chapel Hill, Chapel Hill, NC, USA; Phone: +86 13560294997 Email: jdtucker@med.unc.edu

${ }^{7}$ Sidaction, 228 rue Faubourg Saint-Martin, 75010 Paris, France; +33 15326 49 61; Email:

v.noseda@gmail.com

${ }^{8}$ Oswaldo Cruz Foundation, Evandro Chagas National Institute of Infectious Diseases and Bio-Manguinhos, Avenida Brazil 4365 Manguinhos, CEP 21040-360, Rio de Janeiro, Brazil; Phone: 3865-9559; Email: cristina.possas@ini.fiocruz.br
\end{abstract}

\title{
Correspondence and reprints:
}

Cynthia I Grossman, PhD

Division of AIDS Research, NIMH

5601 Fishers Lane Room 9G19, MSC 9831

Bethesda, MD 20892

Phone: (240) 627-3868

Fax: (240) 627-3107

E-mail: grossmanc@mail.nih.gov

Keywords: HIV cure, multi-disciplinary, ethics, public understanding of science, costeffectiveness

\section{Abstract}

The quest for a cure for HIV remains a timely and key challenge for the HIV research community. Despite significant scientific advances, current HIV therapy regimens do not 
completely eliminate the negative impact of HIV on the immune system; and the economic impact of treating all people infected with HIV globally, for the duration of their lifetimes, presents significant challenges. This article discusses, from a multi-disciplinary approach, critical social, behavioral, ethical, and economic issues permeating the HIV cure research agenda. As part of a search for an HIV cure, both the perspective of patients/participants and clinical researchers should be taken into account. In addition, continued efforts should be made to involve and educate the broader community. 


\section{HIV cure research background}

Significant advances in the optimization of HIV treatment and care have led to life expectancies of HIV-infected individuals similar to uninfected individuals [1 - 3]. However, current HIV therapy regimens can induce life-long toxicities and do not completely eliminate the negative impact that HIV has on systemic inflammation and the immune system [4, 5]. Moreover, the sheer economic impact of treating all people living with HIV globally for the duration of their lifetimes presents significant economic challenges. As such, the quest for a cure for HIV remains a timely and key challenge for the HIV research community.

The past five years have been marked by a growing interest and engagement in HIV cure research and a rapidly expanding literature on the topic. While the majority of HIV cure research has been in the domain of biomedical, translational, and clinical sciences, the prospect of an HIV cure generates implications that go well beyond these disciplines. The broader HIV cure research agenda must address the perceptions, beliefs and expectations of both patients/participants and clinical researchers, attend to issues of equity and access to HIV cure studies and future interventions, and engage and educate other stakeholders, the broader community and the general public as novel strategies progress to opportunities for implementation (Figure 1).

\section{Basic and clinical science of HIV cure research}

Biomedical science clearly has demonstrated that HIV infects CD4+ T cells and integrates into the host genome, with infected cells generally activated and killed by the immune system. A small number of cells do not die, but instead revert to a resting state [6]. These infected resting CD4+ $\mathrm{T}$ cells contain latent provirus, which can live indefinitely undetected by host immunity or antiretroviral therapy (ART). In addition, these cells can be reactivated to produce infectious virus and thereby represent a major obstacle to HIV eradication efforts [7]. Other HIV-infected 
cells such as tissue macrophages, astrocytes, and dendritic cells residing in different anatomic compartments (gut mucosa, genital tract, lymph nodes, and the central nervous system) also contribute to the persistent latent reservoir, rendering viral control or elimination quite complex [8 - 10]. Despite these challenges, HIV cure researchers are developing tools to better understand how viral reservoirs are established and maintained in order to control reactivation or eliminate viral reservoirs altogether.

As biomedical research has evolved, so has the definition of HIV cure, which currently includes two primary concepts: eradication (also termed "sterilizing cure"), in which HIV is completely eliminated from the body, and remission, in which some HIV remains present but is controlled below detection in the blood without antiretroviral therapy [11] and without provoking any adverse effects in the body. Evidence that HIV remission can be achieved was demonstrated from clinical research with a sub-group of patients in the VISCONTI cohort in France, referred to as post-treatment controllers, who were treated very early following HIV acquisition and were subsequently able to control the virus without the need for continuous therapy [12]. A more recent case, presented at the $8^{\text {th }}$ IAS conference on HIV pathogenesis, treatment and prevention, includes an adolescent who was treated soon after birth until age six and has remained virally suppressed in the absence of $A R T$ for the past 12 years (http://www.iasociety.org/Web/WebContent/File/HIV_Cure_Symposium_2015/Day2/Abstracts/O A4_4_LB.pdf). Currently, evidence for eradication has been seen in only one individual, Timothy Ray Brown (also known as "The Berlin Patient"), who, in the course of allogeneic bone marrow transplant therapy precipitated by acute myeloid leukemia, was transplanted with cells resistant to HIV. All evidence to date indicates that HIV has been eliminated from his body with no recurrence in the absence of ART [13].In addition to these results, increased understanding of HIV latency and reservoirs has opened up new avenues for clinical studies, which now include a range of approaches, including interventions with one or more drugs, immune-based therapies, 
latency-reversing agents, early treatment of HIV as close to infection as possible, gene therapy, stem cell transplantation or a combination of these. At this early stage of HIV cure research, much uncertainty exists about the optimal strategies and types of interventions, as many of the ongoing or planned clinical studies involve the use of new therapeutic agents and novel strategies with unknown toxicities and side effects, as well as having the potential for suboptimal outcomes in early-phase experiments.

The prospect of life-long ART and the difficulty in adhering to treatment makes HIV cure a desirable goal for the pediatric population. Babies born to infected mothers are of key interest with HIV cure studies because of their favorable profile for achieving restricted HIV reservoir. Immediate ART close to the onset of HIV infection at birth is the standard of care worldwide, providing an opportunity for prompt control of HIV spreading. Babies have few memory CD4+ T cells that are prime targets for HIV. As a result, early treated infants may possess a high likelihood of reaching HIV remission. However, the limited knowledge on the mechanisms of latency in infants and children hinders the development of cure interventions for this population. Further challenges are the need for virologic/immunologic assays with small blood volume requirement and the lack of non-invasive methods to investigate HIV in tissues. Importantly, the decision for children to participate in trials is made by proxies, and potential risks and gains must be carefully balanced [14].

As this experimental research advances toward testing new clinical strategies, critical research questions emerge that must be considered from an integrated multi-disciplinary approach that includes social and behavioral scientists, ethicists, and modeling experts in concert with biomedical and clinical researchers, and that acknowledges and optimizes the interdependency of researchers, patients, providers, communities, and other stakeholders. 


\section{Patient perspectives}

As of early 2015, there were more than 100 HIV cure-related clinical studies being conducted or completed worldwide (http://www.treatmentactiongroup.org/cure/trials). An important component of these studies is participant safety, as they include interruption of antiretroviral therapy and/or invasive procedures to investigate critical endpoints, such as decreased reservoir size or viremic control. The planning phase for future clinical studies provides an opportunity to better understand how study participants actually comprehend and consider the risks of the clinical investigation, the experimental nature of early studies, and cure-related terminology (e.g. cure, remission, eradication, and analytic treatment interruption). Behavioral and social science research strategies allow for HIV cure-related research to place the needs and perspectives of people living with HIV at the center of the progression of clinical studies and investigate participant motivations and decision-making processes related to preferences for or participation in different types of intervention studies with various potential outcomes $[15,16]$.

While the field is new, a series of qualitative and quantitative studies have provided early findings in this regard. For example, a survey conducted among over 450 people living with HIV in the Netherlands suggested that no longer being able to transmit HIV to sexual partners was a more desirable outcome than the opportunity for the participant to interrupt ART alone (http://www.iasociety.org/Portals/0/Files/Cure/2012_Symposium/Verdult.pdf). A study of 29 Chinese patients not enrolled in HIV cure studies showed that HIV cure research generated hope for both physical well-being and social standing [17]. A survey among 20 participants who took part in a 14-day study involving serial doses of the histone deacetylase inhibitor (HDACi) vorinostat in Australia [18] found that "stopping HIV transmission to others" was the highest hypothetical benefit of study participation while "stopping doctor visits to monitor HIV" was ranked last. A more extensive online survey conducted in the United States among 2,094 
people living with HIV revealed a high willingness to participate in HIV cure studies involving treatment interruption [19].

Qualitative evidence from the Food and Drug Administration (FDA) initiative on patient-focused drug development for HIV cure research, which resulted in the release of the Voice of the Patient report, demonstrated that individuals living with HIV join HIV cure studies for a variety reasons, including altruism and the desire for personal benefits (http://www.fda.gov/ForIndustry/UserFees/PrescriptionDrugUserFee/ucm348598.htm). These studies and reports focus on the perspectives and preferences of participants and people living with HIV not on the real-time decision-making that occurs as part of participation in a clinical study. In this case, there is a gap in the research that describes this decision-making process and links it to participant behaviors (e.g. enrollment, retention and HIV disclosure). There is also a gap in the inclusion of the perspectives of pregnant women living with HIV and possible participation of their infants in HIV cure studies. There are on-going studies in the United States and other countries investigating willingness to participate in HIV cure-related research, but additional studies are needed.

There are other aspects of the lives of people living with HIV that may affect their basic motivation and willingness to participate in (and advocate for) cure research that may not be readily apparent to basic and clinical scientists. For example, one of the legacies of the HIV/AIDS epidemic (as it is understood and discussed by social scientists), is that of the construction and incorporation of an HIV-positive identity, which has been stigmatized in some contexts and valorized in others [20]. For decades, people living with HIV have been encouraged to 'come out' as HIV-positive, embrace it as part of their identity, and use it as a primary advocacy tool. At the same time that stigma and discrimination makes that process challenging, some groups are encouraging serostatus disclosure as a way to reduce stigma and 
discrimination through advocacy. Indeed, HIV-positive identity has been effectively institutionalized through such documents as the Denver Principles and Greater Involvement of People Living with AIDS (GIPA), as well as membership societies such as the National Association of People Living With AIDS (NAPWA), Global Network for and by People Living with HIV (GNP+), International Community of Women Living with HIV/AIDS (ICW), and HIV Positive Women's Network (PWN). For people living with HIV, the extent to which HIV cure research calls into question core identity and membership status within the AIDS community, serves as a potential to reduce the negative impact of HIV stigma and discrimination, and/or raises issues of the construction and maintenance of different HIV-related identities is an important social science question in and of itself, but may also affect the trajectory of HIV cure-related clinical research.

An in-depth understanding of patient/participant motivations, preferences, beliefs, expectations, identities, hopes, and decision-making process will ensure effective patient education, position patients to understand and interpret study outcomes, and facilitate the design and conduct of future HIV cure clinical studies.

\section{Patient/study participant: clinician/researcher relationships}

HIV clinicians play a key role in organizing the clinical care of patients, in addition to serving as principal investigators on major clinical research studies. It is important to understand how this dual role and relationship may influence various aspects of HIV cure research. This includes clinician and researcher attitudes about who should participate in what kind of research. Currently, most HIV cure studies represent proof-of-concept activities with little-to-no potential clinical benefit and high risks for the study participant [21, 22]. From the perspective of the clinical researcher, individuals who are identified early in their infection and are virally suppressed with current therapies may be the best candidates for early proof-of-concept 
studies, while women living with HIV who are also of childbearing potential may be excluded because of perceived safety concerns. An additional consideration is whether participation in one study precludes participation in subsequent studies and, if so, how patients and clinical researchers decide on participation or non-participation. Other aspects of the clinician/researcher and patient/participant relationship include the informed consent process, the potential for therapeutic - or curative - misconception (i.e., when a participant does not fully understand the boundaries between standard clinical care and experimental clinical research) [23 - 25], and the management of the expectations of both parties.

These concerns and considerations argue for integrating patient-centered and shared decisionmaking approaches into HIV cure research, to ensure a meaningful exchange of knowledge between patients/participants and clinicians/researchers in an act of collaborative knowledge building [26]. To date, the majority of survey research has focused on the patient/participant, highlighting a need for additional inquiry into the perspectives of clinicians and clinical researchers.

\section{Community, policy-maker, and funder perspectives}

Given the historical significance and global impact of the HIV epidemic, stakeholders in HIV cure-related research go beyond individual patients/participants and providers/researchers, to include communities, policy-makers, and funders [27]. There are a host of relevant research questions related to these broader stakeholders, including how individuals and communities understand the notion of cure itself, as well as the science behind it, and how this affects responses to and support for various HIV cure research approaches.

A public understanding of science (PUS) approach is helpful in this regard, by unpacking how the general public understands and uses different knowledge in their lives-how people create 
meaning from scientific findings and incorporate it into their everyday lives and decision-making, and to what effect [28]. The PUS approach includes, but is not limited to, community engagement in research. It goes beyond a focus on recruitment and retention in clinical studies, or on health or HIV literacy. Rather, it investigates how the general public comprehends and understands what science is and does, including the way it is conducted and the interpretation of its results. It also includes analysis of who is conducting and supporting the research, and the reality of their stated or perceived interests.

With respect to HIV cure research specifically, it is important to consider how individuals and communities understand basic terminology (e.g., cure, remission, and eradication) [29] and how they weigh and balance competing notions of cure coming from different 'experts', including previous or alternative notions of HIV cure that have been discounted by scientists [30]. Additionally, it is important to know how the social construction of cure, including how cure is communicated affects what is and is not studied, as well as what is or is not taken up. The social construction and how it is communicated have a link to policy and advocacy around HIV cure research.

Policy-makers and funders are key stakeholders in HIV cure-related research. As they make difficult resource allocation decisions in an environment of increasingly constrained resources, they must weigh investing in cure research and its eventual implementation - something that is emergent and still aspirational - against investing in other approaches to ending the AIDS pandemic such as HIV vaccine research or enhancing implementation of methods that already exist for preventing HIV infections, such as condom use, pre-exposure prophylaxis (PrEP), harm reduction programs for drug users, and ART use among people living with HIV. Particularly in the early phases of cure research, where uncertainty prevails, these kinds of decisions—which are relevant to both resource-rich and resource-poor countries and settings-are best informed 
by input from ethics analyses focused on issues of safety and equity, and by social and economic research employing such methods as scenario-building, cost-effectiveness analysis, and mathematical modeling.

Cost-effectiveness analysis of current therapeutic or preventive interventions is an important tool for decision-making, recognizing its complexities and limitations. One example of a recent model by Sax and colleagues found that depending on efficacy, relapse rate and cost some HIV cure interventions were superior in terms of cost-savings [31]. These types of modeling studies may prove useful in guiding the desired performance characteristics of cure strategies. This approach requires selecting adequate measures of effects, which depend on the nature of the intervention being considered, and monetizing health effects, which can be sometimes difficult and/or inappropriate. For HIV cure strategies and interventions still in development, it is important to underline the limitations of cost-effectiveness analysis due to the uncertainty in HIV cure research and the potential impact of new interventions. For this reason, other strategies such as mathematical models that include accounting for uncertainty will need to be explored, for example, Value of Information analysis (VOI) which refers to the amount a decision-maker would be willing to pay for information prior to making a decision [32, 33]. These kinds of analytic strategies are detailed by Freedberg and colleagues in reference to HIV cure research, yet economics related research in HIV cure in general has yet to be fully undertaken [34].

Once an effective cure strategy is identified, implementation science will be key to identifying how best to operationalize it in the context of various health care systems. Recent experience with the development of a cure for hepatitis $\mathrm{C}$ virus (HCV) illustrates the importance of integrating these perspectives early on to anticipate and, hopefully, mitigate the possibility of a high-cost intervention exacerbating inequalities in access and resultant health disparities across populations. Even though HIV cure strategies may not be readily accessible and scalable right 
now, planning for this development is critical to the degree possible. For example, developing countries could improve their systems of early HIV diagnosis and access to ART which would benefit patients, the health care system and potentially further HIV cure research in these areas of the world. In addition to the health and prevention benefits, keeping patients virally suppressed limits the reservoir size which could place the individuals at an advantage to respond to future cure interventions.

\section{Concluding remarks}

HIV cure-related research (like most public health science) is not something that occurs in a laboratory vacuum, but rather is affected by the cultures and contexts surrounding it, and the inputs and actions of various stakeholders. Patients, study participants, providers, researchers, communities, policy-makers, and funders are key stakeholders in the pursuit of an HIV cure, and it is imperative to study the nature of their involvement, perspectives, and decision-making as these influence all phases of HIV cure research, as well as their impact on proposed

outcomes. Social, behavioral, and economic research is necessary to ensure that the new HIV cure strategies and products, when available, will be scalable and accessible to all populations (see Outstanding Questions). The creation of academic networks, such as the NIH-funded HIV/AIDS Clinical Trials Networks, to bring together representatives of all relevant disciplines including basic sciences, translational research, clinical research, ethics, social medicine, anthropology, economics, health policy - has fostered fertile ground for cross-dialogue and interaction, and these networks should be further encouraged and strengthened. Equally important is ongoing dialogue between the public and private sectors, which to date, has been instrumental in promoting and accelerating HIV cure research. To ensure that any HIV cure strategy will be available, scalable and accessible once proven effective, it will be crucial to understand how governments and other payers incorporate and disseminate new interventions and technologies into their decision-making and how this affects access. It will be also crucial to 
understand the role and potential of media globally and locally to influence public engagement in HIV cure research and the implementation of its findings. As science advances toward a cure for HIV, it is imperative to integrate multiple disciplines in novel, compelling, and productive ways to ensure its ethical and effective application and its promise for contributing to the transformation of the HIV/AIDS pandemic.

\section{Acknowledgments:}

The contributions of the International AIDS Society (IAS) Towards an HIV Cure Working Groups, especially the Psychosocial, Cost-Effectiveness and Ethics Working Groups are acknowledged as critical in the development of the content found in this manuscript. The IAS Towards an HIV Cure team and Rosanne Lamplough are acknowledged for their facilitation of the meetings and discussions that led to this manuscript. KD is supported by NIAID U19 AI096113 (Collaboratory of AIDS Researchers for Eradication; PI: David M. Margolis). JT is supported by NIAID P30-AI50410, NIAID R01A108366-01. The content is solely the responsibility of the authors and does not necessarily represent the official views of the National Institutes of Health, the U.S. Army or the U.S. Department of Defense. All authors do not have any related conflicts of interest. 


\section{REFERENCES}

1. Nakagawa F. et al. (2012) Projected life expectancy of people with HIV according to timing of diagnosis. AIDS 26(3):335-343

2. Boulle A. et al. (2014) Mortality in patients with HIV-1 infection starting antiretroviral therapy in South Africa, Europe, or North America: a collaborative analysis of prospective studies. PLoS Medicine;11(9):e1001718.

3. Samji H. et al. (2013) Closing the gap: increases in life expectancy among treated HIVpositive individuals in the United States and Canada. PloS One 8(12):e81355..

4. Valdez H. et al. (2002) Limited immune restoration after 3 years ' suppression of HIV-1 replication in patients with moderately advanced disease. AIDS16:1859-1866.

5. Lederman M.M. et al. (2011) Immunologic failure despite suppressive antiretroviral therapy is related to activation and turnover of memory CD4 cells. The Journal of Infectious Diseases 204(8):1217-1226.

6. Chomont N. et al. (2009) HIV reservoir size and persistence are driven by T cell survival and homeostatic proliferation. Nature Medicine 15(8):893-900.

7. Buzon M.J. et al. (2014) Long-term antiretroviral treatment initiated in primary HIV-1 infection affects the size, composition and decay kinetics of the reservoir of HIV-1 infected CD4 T cells. Journal of Virology; 88(17):10056-10065.

8. Deeks S.G. et al. (2013) The end of AIDS: HIV infection as a chronic disease. Lancet 382(9903):1525-1533.

9. Deeks S.G. et al. (2012) Towards an HIV cure: a global scientific strategy. Nature Reviews Immunology 12(8):607-614.

10. Ho Y-C, et al. (2013) Replication-competent noninduced proviruses in the latent reservoir increase barrier to HIV-1 cure. Cell 155(3):540-551.

11. Fauci A.S. et al. (2013) HIV-AIDS: much accomplished, much to do. Nature Immunology 14(11):1104-1107.

12. Sáez-Cirión A. et al. (2013) Post-treatment HIV-1 controllers with a long-term virological remission after the interruption of early initiated antiretroviral therapy ANRS VISCONTI study. PLoS Pathogens 9(3):e1003211.

13. Allers K. et al. (2011) Evidence for the cure of HIV infection by CCR5 $\Delta 32 / \Delta 32$ stem cell transplantation. Blood 117(10):2791-2799.

14. Rossouw T.M., et al. (2015). Ethics of HIV research in children. Lancet Infect Dis 15(6):634-5.

15. Tucker J.D. et al. (2014) Social and ethical implications of HIV cure research. AIDS 28(9):1247-1250.

16. Dubé K. et al. (2015). Participation in HIV cure-related research: a scoping review of the proxy literature and implications for future research. Journal of Virus Eradication 1; e14 - e20.

17. Chu C.E. et al. (2015) Exploring the social meaning of curing HIV: a qualitative study of people who inject drugs in Guangzhou, China. AIDS Research and Human Retroviruses 31(1):78-84.

18. McMahon J.H. et al. (2015) Experiences and expectations of participants completing an HIV cure focused clinical trial. AIDS 29(2):248-250..

19. Arnold M. et al. (2015) Recruitment and ethical considerations in HIV cure trials requiring treatment interruption. Journal of Virus Eradication1(January):43-48.

20. Baumgartner L. et al. (2009) Accepting being poz: the incorporation of the HIV identity into the self. Qual Health Research 19(12):1730-1743.

21. Lo B. et al. (2013) Ethical considerations in HIV cure research: points to consider. Current Opinion in HIV and AIDS 8(3):243-249. 
22. Dubé K. et al. (2014) Framing expectations in early HIV cure research. Trends in Microbiology 22(10):547-549.

23. Henderson G. E. et al. (2006) Therapeutic misconception in early phase gene transfer trials. Social Science \& Medicine 62(1):239-253.

24. Peay H. et al. (2015) What motivates participation in HIV cure trials? A call for real-time assessment to improve informed consent. Journal of Virus Eradication 1:51-53.

25. Rich Z. et al. (2015) Physician perceptions of HIV cure in China: A mixed methods review and implications for HIV cure research. Asian Pacific Journal of Tropical Disease. 5(9): 687-690.

26. Kukla R. (2007) How do patients know. Hastings Center Report 5(5):27-35.

27. Lo Y. et al. (2015) Stakeholder engagement in HIV cure research: lessons learned from other HIV interventions and the way forward. AIDS Patient Care and STDs 29:1-11.

28. Bauer M.W. et al. (2007) What can we learn from 25 years of PUS survey research? Liberating and expanding the agenda. Public Understanding of Science 16(1):79-95.

29. Tucker J. et al. (2014) Words matter: discussing research towards an HIV cure in research and clinical contexts. JAIDS JAcquir Immune Defic Syndr 67(3):110-111.

30. Rennie S. et al. (2015) The ethics of talking about "HIV cure". BMC Medical Ethics 16:18.

31. Sax P.E. et al. (2014) HIV cure strategies: how good must they be to improve on current antiretroviral therapy? PLoS One 9(11): e 113031.

32. Willan A.R. et al. (2012) Value of information methods for planning and analyzing clinical studies optimize decision making and research planning. J Clin Epidemiol 65(8):870-876.

33. Wilson E. (2015) A Practical Guide to Value of Information Analysis. PharmacoEconomics 33(2):105-121.

34. Freedberg K (2015). The HIV cure research agenda: the role of mathematical modeling and cost-effectiveness analysis. Journal of Virus Eradication 1, 245-249 
Figure 1. Critical questions to address in the integration of social science in the HIV cure research agenda. 


\section{Outstanding Questions}

- What is the role of social science (e.g. social and behavioral research) in early basic research? What are the preferences, beliefs and hopes of patients, research participants and clinical researchers for specific HIV cure studies? What, if any, is the influence of social and contextual factors on biomarkers used in HIV cure studies? What influences patients' willingness to participate in different HIV cure studies? These questions could be studied using mixed methods research as well as inclusion of social and behavioral surveys.

- What are the social and ethical considerations in early clinical research? What is the relationship of informed consent procedures and therapeutic and curative misconception? What is the shared-decision making process for different HIV cure studies? This could be studied using empirical research on informed consent and examing social science of decision-making frameworks.

- What are the critical aspects of community and stakeholder engagement? What is the perception of the community and their understanding of the scientific process related to HIV cure? These questions could be studied by looking at public understanding of science (PUS) and empirical research on community engagement.

- What is needed to ensure the strategies be scalable and effective in the real world? What is the early understanding of the potential cost-effectiveness of an HIV cure? What is needed regarding readiness for potential implementation, particularly regarding health systems and policy research? Further work could be done with modeling, health services research frameworks for preparedness, and through policy research. 


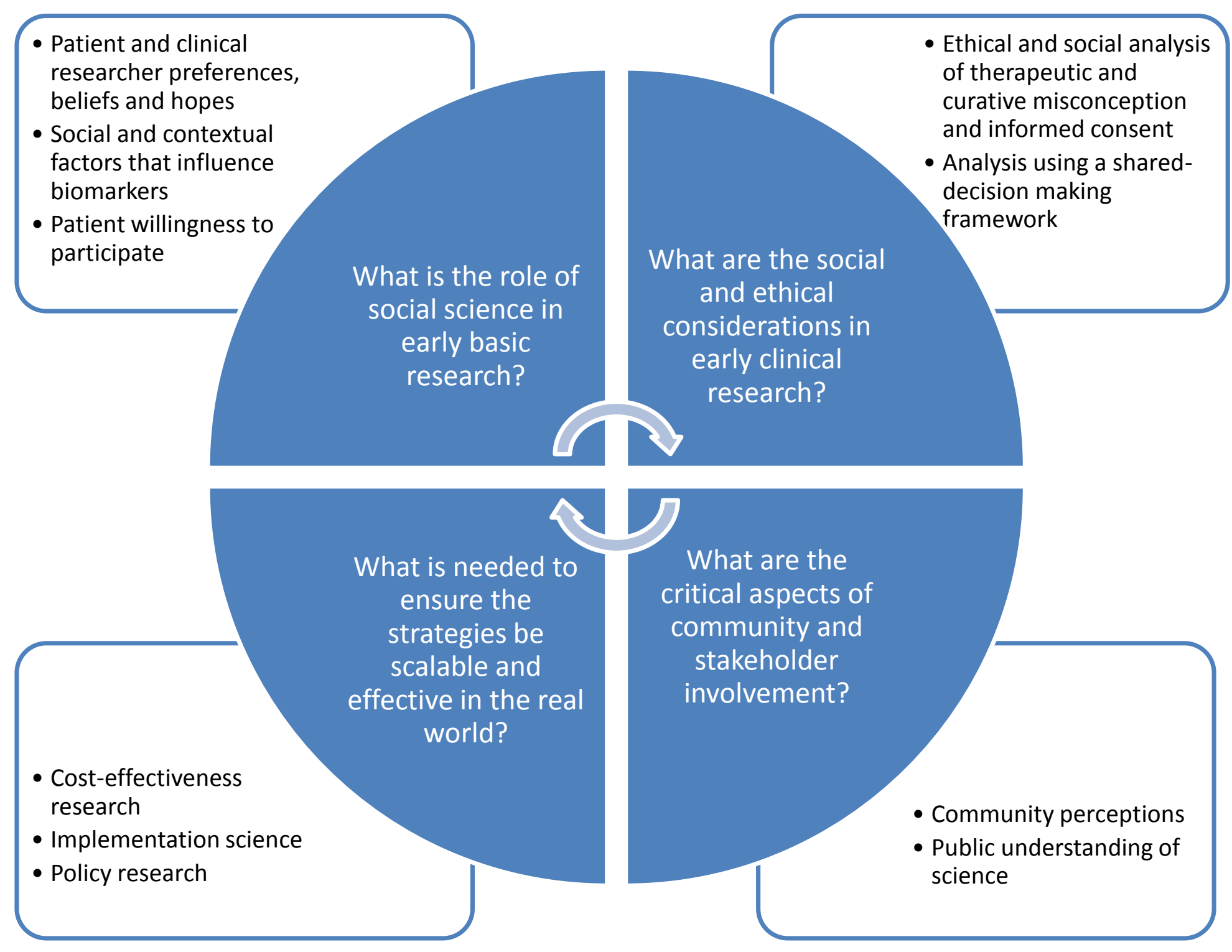

